\title{
Carnets
}

Revue électronique d'études françaises de l'APEF

Deuxième série - 5 | 2015

Imaginaires de guerre et autres conflits

\section{La guerre, la mémoire et les récits de vie}

\section{Gökçe Bayindir Goularas et Ayşe Betül Nuhoğlu}

\section{(2) OpenEdition}

\section{Journals}

Édition électronique

URL : http://journals.openedition.org/carnets/407

DOI : $10.4000 /$ carnets. 407

ISSN : 1646-7698

Éditeur

APEF

\section{Référence électronique}

Gökçe Bayindir Goularas et Ayşe Betül Nuhoğlu, «La guerre, la mémoire et les récits de vie », Carnets [En ligne], Deuxième série - 5 | 2015, mis en ligne le 30 novembre 2015, consulté le 21 avril 2019. URL : http://journals.openedition.org/carnets/407 ; DOI : 10.4000/carnets.407

Ce document a été généré automatiquement le 21 avril 2019.

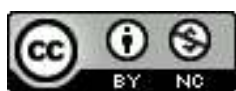

Carnets est mis à disposition selon les termes de la licence Creative Commons - Atribution - Pas d'utilisation commerciale 4.0 International. 


\title{
La guerre, la mémoire et les récits de vie
}

\author{
Gökçe Bayindir Goularas et Ayşe Betül Nuhoğlu
}

\section{Introduction}

1 En 1922, la guerre appelée la guerre d'indépendance turque par les Turcs, la guerre grécoturque par les Grecs, s'achevait. Avec la défaite grecque contre la victoire turque, la nouvelle République turque concrétisait sa fondation. Depuis les guerres balkaniques de 1912-1913, les migrations massives des populations musulmanes de la Grèce vers l'Anatolie et des populations grecques orthodoxes de l'Anatolie et de la Thrace orientale vers la Grèce ne cessaient de s'accroître. Le tracé définitif de la frontière en 1923 entre les deux États laissait une population musulmane sur les territoires de la Grèce, lesquels furent sous la domination ottomane jusqu'à la perte territoriale de l'Empire en Roumélie. Les nouvelles frontières de l'État turc englobaient une population grecque orthodoxe, qui, auparavant était considérée comme une partie de la population non-musulmane de l'État ottoman. Dans ce contexte de redéfinition des frontières nationales et de formation des États-nations, l'homogénéisation de la population souhaitée par le côté grec et le côté turc a été réalisée par la signature de la Convention concernant l'échange des populations grecque et turque, le 30 janvier 1923. Ainsi, 456720 Musulmans de Grèce s'installèrent en Turquie (Belli, 2006 : 95-96) et plus de 200000 Grecs orthodoxes d'Anatolie en Grèce (plus de 1200000 Grecs avaient déjà migré vers la Grèce avant l'an 1923).

2 Les années de guerre, la période des migrations, la misère et la pauvreté de l'époque ont rapidement trouvé place dans l'historiographie et la littérature de la Grèce et de la Turquie. Par contre, le reflet de la mémoire de la guerre et de la migration dans les romans et récits de vie dans les deux pays est difficile à comparer. En Grèce, il existe une bibliographie très riche concernant la période d'avant et d'après 1923, dont les témoignages et les récits de vie constituent les sources principales. Les mémoires des années de guerre, la migration des Grecs d'Anatolie vers la Grèce et l'échange des populations ont occupé une place primordiale dans les publications de l'époque dès le 
lendemain des événements en question. Les associations et les centres des réfugiés et des échangés grecs ont joué un rôle prépondérant dans l'enrichissement de cette littérature spécifique (Millas, 2005 : 418). En Turquie, malgré l'existence d'une riche bibliographie sur l'histoire de la guerre, la mémoire de la guerre et les migrations liées à cette période précise, notamment l'échange des populations, n'ont pas trouvé une place considérable dans la littérature turque jusqu'aux années 1990. Il est donc possible de parler d'une différence de l'importance accordée dans le récit de la période concernée laquelle est particulièrement liée à la différence dans la perception des événements par les Grecs et les Turcs, les premiers étant les perdants et les derniers les gagnants de la guerre. Pour la Grèce, ses pertes territoriales en Anatolie signifiaient la fin de la Grande Idée, alors que pour la Turquie, c'était le début d'une nouvelle époque, la formation de la République turque (Millas, 2005: 419). Le reflet du traumatisme de la guerre dans la littérature grecque était une conséquence naturelle de la guerre. Tandis qu'en Turquie, les années de guerre et par conséquent l'échange des populations étant inévitables pour la formation de l'État-nation turc, il n'était pas nécessaire de s'en souvenir et de parler du passé des territoires autres que ceux d'Anatolie. En Turquie, à partir du milieu des années 1990, le silence du côté associatif, du côté de la recherche et de la publication des récits de vie et des témoignages a cédé la place à une explosion des recherches sur cette période de l'Histoire, à la création de la première association des échangés et à la multiplication des publications scientifiques et littéraires.

3 Le but de cet article est d'analyser les récits de vie des Grecs et des Turcs, qui ont témoigné des années des guerres et de la migration obligatoire qui les a suivies, afin de saisir les similarités et/ou les différences entre la représentation écrite de cette période conflictuelle en Turquie et en Grèce. Pour réaliser ce travail, nous avons identifié 114 ouvrages turcs et 41 livres grecs traduits en Turc, tous étant des romans ou des recueils de témoignages concernant des récits de vie de l'époque de la guerre et des migrations. La plupart des livres grecs sont traduits en turc après les années 1990 mais parus en Grèce à partir de 1960. Quant aux livres turcs, une partie d'entre eux est parue dans les années 1990 mais une majorité considérable date de la dernière décennie.

4 Ce travail a été réalisé grâce à l'étude détaillée de 60 ouvrages (30 grecs et 30 turcs) en se référant aux citations de quatorze romans et livres de témoignages (dont sept grecs et sept turcs et cinq livres de témoignages contre neuf romans) sélectionnés. Dans tous les livres analysés, les personnages principaux ont vécu pendant ces années de guerre (sur 142 témoignages étudiés 136 appartiennent à la première génération). Nous avons pu assurer un équilibre entre le nombre de caractères féminins et masculins dans les romans. Par contre, dans les témoignages nous avons analysé les récits de 89 hommes contre 53 femmes, dont une grande partie étaient des habitants des régions rurales ou de petites villes et s'occupaient principalement de l'agriculture et de l'élevage. Toutes les citations utilisées dans ce travail sont des traductions libres des auteurs de cet article.

\section{La mémoire de la guerre}

5 La mémoire de la guerre dans la littérature grecque et dans la littérature turque présente plus de différences que de similarités parmi lesquelles la perception de la guerre, la description de la cohabitation des deux communautés ainsi que l'attribution de la responsabilité de la guerre attirent particulièrement l'attention. 
6 Avant tout, concernant la perception de la guerre elle-même, il suffit d'une première lecture des romans et des récits de vie pour saisir que la guerre était plus destructrice pour les habitants grecs d'Anatolie que pour les habitants turcs de Grèce. Le fait que la guerre se déroulait activement sur les territoires anatoliens peut être considéré comme argument principal mais ce seul fait n'est pas suffisant pour expliquer la différence de perception de la guerre les ouvrages grecs et turcs. Car les Turcs de Grèce, même s'ils ne se sont pas retrouvés dans les champs de bataille comme en Anatolie, ont vécu la montée de tension politique et sociale en raison de la guerre et des violences des bandes armées grecques. De plus, les deux peuples ont dû quitter leurs terres natales en raison de la question de sécurité, des actes violents ou des pressions des armées adversaires et de la décision de l'échange obligatoire des populations. Néanmoins, la défaite de l'armée grecque en Asie mineure signifiant la fin de la Grande Idée grecque contrairement à la victoire turque en Anatolie et la formation d'un État-nation turc comme il a été défini par le Pacte national turc à partir de l'an 1919, se reflètent différemment dans la littérature des deux pays. La littérature grecque donne souvent place aux mauvais souvenirs et commentaires de la guerre alors même que, la littérature turque, nous présente une description des évènements catastrophiques avec pour l'année 1922. L'emploi fréquent du mot «catastrophe » dans les ouvrages grecs pour l'an 1922, l'année de la défaite grecque en Asie mineure n'est donc pas un hasard: « la seule chose dont je suis sure, c'est que nous sommes en l'an 1922, une période de contes a pris fin et les peines et la catastrophe ont débuté » (Molivyatis, 2005 : 22). Dans la même logique, la guerre entre les Grecs et les Turcs est décrite dans la plupart des romans et des récits de vie grecs comme une mobilisation turque pour faire partir les Grecs des territoires anatoliens: «Mois de janvier de 1922. La mobilisation des Turcs en vue de chasser les Grecs d'Asie mineure est son apogée » (Tsolakidis, $2007: 82$ ). «C'était une catastrophe et une destruction totale. Nous (les Grecs) n'étions que des objets sans valeurs, des esclaves qui n'ont pas d'espoir de vie» (Molivyatis, 2005 : 32). Dans la plupart des livres turcs, la même période est décrite comme celle pendant laquelle les Turcs de Grèce apprenaient plus tardivement ce qui se passait en Anatolie :

La guerre balkanique avait débuté. Ses échos étaient arrivés jusqu’à la région de la Mer noire. Dimitro parlait de cette situation et partageait ses sentiments avec ses amis turcs...Ils (les Grecs et les Turcs) vivaient dans une ambiance de paix avec une harmonie de fraternité, en croyant au même Dieu (Andreadis, 1999 : 26).

7 La raison de la violence des Grecs contre les Turcs est souvent décrite comme une conséquence de la guerre en Anatolie : « La nouvelle de la défaite de l'armée grecque a été diffusée en Anatolie et un peu plus tard, celle de la libération d'Izmir. Les Grecs de notre région avaient peur de ces nouvelles» (Özsoy, 2003: 172). Avec la dégradation de la situation et les rumeurs qui circulaient à propos de la guerre, la peur et l'inquiétude envahissaient le quotidien de deux peuples :

Malheureusement, les conflits et les assassinats en Crète se multipliaient. Nous avions entendu que (par les Grecs d'Anatolie) Mustafa Kemal Pacha avait chassé les Grecs jusqu'à İzmir, et qu'il nettoyait l'Anatolie (des Grecs). Avec ces nouvelles et les provocations des Grecs d'Anatolie migrés vers la Crète, ils (les Grecs de Crète) avaient commencé à tuer notre peuple (Turc) et à battre à mort ceux qu'ils ne tuaient pas. Les actes de violence calmés pour une période, étaient de nouveau multipliés (Yorulmaz, $2004: 120$ ). 
En deuxième lieu, par rapport aux livres grecs, les livres turcs attirent plus souvent l'attention sur la cohabitation pacifique des communautés grecques et turques avant la guerre mais aussi malgré la guerre :

«Qu'il soit Turc ou Grec, c'est toujours le peuple, l'homme qui souffre mon enfant. Sans les jeux de pouvoir des dirigeants qui essayent de garder leur place (au pouvoir), nous, c'est-à-dire le peuple, nous pouvons continuer de vivre ensemble et en paix » (parole d'une femme grecque à un jeune turc, Yorulmaz, 2004 : 95).

Cette cohabitation pacifique est assurée par le bon voisinage des deux communautés mais aussi par la bonne entente des chefs spirituels des communautés musulmane et chrétienne :

« Nous habitions (les Musulmans et les Chrétiens) dans le même quartier. Les seuls endroits qui nous séparaient étaient la mosquée et l'église. Nos peuples (les Musulmans et les Chrétiens) ont vécu ensemble en paix et heureux pendant que l'Imam et le Pope du village se saluaient et discutaient » (Yorulmaz, $2004: 20$ ).

Dernièrement, en ce qui concerne les responsables de la guerre, dans les livres grecs et turcs, nous remarquons deux explications différentes: Atatürk et ses troupes pour les Grecs ou les puissances étrangères; Venizélos et l'armée grecque et plutôt les Grecs d'Anatolie migrés en Grèce selon les Turcs. Les ouvrages grecs soulignent assez souvent le fait que les Turcs d'Anatolie n'ont pas essayé de faire souffrir les Grecs d'Anatolie, c'est Mustafa Kemal et ses troupes, décrits en tant qu'ennemis des Grecs qui sont responsables des souffrances des Grecs :

Les Turcs de Simav étaient des gens tranquilles et modestes. Nous nous entendions bien avec eux, nous vivions comme des frères et des sœurs. Mais malheureusement ce sont les hommes violents de Mustafa Kemal qui ont commis ces horreurs (Kücük Asya Araştırmaları Merkezi, 2004 : 252).

11 Dans la plupart des ouvrages grecs, le sentiment d'hostilité contre Mustafa Kemal et ses troupes a été mentionné et considéré comme la principale raison qui empêche l'amitié entre les peuples grec et turc. Mustafa Kemal est vu comme celui qui a comme objectif principal l'anéantissement de la population grecque orthodoxe de la Turquie : «Mustafa Kemal avait décidé de nous anéantir, c'est pour cela qu'ils (Mustafa Kemal et ses troupes) ont envoyé nos hommes en exil au centre de l'Anatolie, en Cappadoce» (Kücük Asya Araştırmaları Merkezi, 2004: 80); "Les Turcs (l'armée) ont utilisé des méthodes diaboliques pour faire disparaître la population de la région (de la Mer noire)» (Andreadis, 1999: 59) ; «Un homme appelé Mustafa Kemal est apparu en Anatolie au milieu de l'an 1919. Ce Mustafa Kemal était le Dieu des Turcs, le Diable des Grecs » (Kücük Asya Araştırmaları Merkezi, 2004 : 80) ; « Leur comportement (de la bande armée turque) était sans pitié, nous avions compris que la disparition de la population grecque était leur but national et sacré » (Molivyatis, $2005:$ 31). D'après l'auteur du roman grec Tolika, la période difficile pour les Grecs de la Mer Noire a commencé avec l'arrivée de Mustafa Kemal à Samsun, une ville importante de la région (Andreadis, 1999:58). Les soldats turcs détruisaient les villages grecs et plusieurs Grecs avaient dû se réfugier dans les montagnes pour se protéger : « Les soldats et les gendarmes sont entrés dans notre village (...) les soldats et les bandes armés ont détruit tous ce que nous possédions » (Andreadis, 1999 : 74-76).

12 Même si dans presque tous les livres grecs l'armée turque et Mustafa Kemal sont les ennemis principaux des Grecs, certains livres attribuent à certaines autres grandes puissances la responsabilité de la mauvaise relation entre les Turcs et les Grecs. À titre d'exemple, d'après Sotiriou, certains grands pays désirant assurer une hégémonie dans 
l'espace géographique géopolitiquement très important de la Grèce et de la Turquie, ont essayé d'empêcher les bonnes relations gréco-turques. Par conséquent, les peuples grec et turc qui vivaient auparavant en paix et en harmonie en partageant pendant des siècles la même géographie et ayant de nombreuses interactions culturelles, sont devenus des ennemis mortels : «Que le Dieu punisse ceux qui font tuer son frère! (...) Les frères, les amis, les compatriotes, une génération entière s'auto-assassine pour rien » (Sotiriou, 2013 : 260). Cette affirmation existe aussi chez d'autres auteurs grecs : "Comment nous allons consolider nos cœurs blessés? Comment nous allons faire croire les grands (grandes puissances) qu'ils n'avaient pas droit à nous utiliser (pour leur propre intérêt) ?» (Molivyatis, 2005 : 161).

13 Quant aux romans et récits de vie turcs, malgré le fait que dans certains Venizélos est qualifié de responsable de la situation conflictuelle, la plupart mentionne l'armée grecque et les Grecs d'Anatolie migrés en Grèce comme les acteurs de la souffrance des Turcs et de la dégradation des relations entre les peuples grec et turc : «En Grèce, l'armée grecque oppressait la population musulmane de la ville (...) les Grecs (soldats) arrachaient les bébés des bras de leurs mères et les tuaient avec des couteaux » (Andıç, 2004 : 16-17).

D'après les témoignages, la période de troubles pour les Turcs vivant sur le sol grec a débuté au moment de l'arrivée des Grecs d'Anatolie en Grèce et de l'apparition des bandes armées grecques : "Nous avions de bonnes relations avec les Grecs de notre village. Mais quand les Grecs d'Anatolie sont venus en Crète, notre ordre a été bouleversé. Ils ont tué nos proches » (Özsoy, $2014: 162)$; « Les Grecs locaux de notre région (Ioannina en Grèce) étaient nos amis, on était comme des frères et des sœurs. Ceux qui étaient violents et hostiles, c'était les Grecs d'Anatolie » (Özsoy, $2003: 37)$; «Les bandes armés (grecques) venaient dans notre village souvent le vendredi (le jour sacré des Musulmans) et volaient les gens qui sortaient de la mosquée. Ils les tuaient aussi parfois » (Özsoy, 2014 : 129).

\section{La guerre et l'appellation de « l'autre »}

15 La guerre influence non seulement la mémoire des personnes concernées mais détermine aussi les noms et adjectifs utilisés pour décrire les membres de l'autre communauté. Les désignations les plus fréquentes dans les livres grecs et turcs sont avant tout liées à l'appartenance ethnique et religieuse des communautés et puis à la perception de l'autre communauté et au type de relations existantes.

Très souvent, l'emploi des mots précisant les identités ethniques (Turc ou Grec) et les identités religieuses (Musulman ou Chrétien) est choisi par rapport au degré et type de relations existantes entre les deux groupes. A titre d'exemple, dans les œuvres des écrivains turcs, soit les « Turcs » d'Anatolie (non pas les Musulmans) sont en cohabitation avec les « Roums » d'Anatolie, soit les «Turcs » de Grèce partagent les mêmes territoires avec les «Grecs ». Cette différence se traduit pour les Turcs, par une distinction entre les Grecs de Grèce et les Grecs d'Anatolie, les premiers étant inconnus et les autres considérés comme compatriotes des Turcs d'Anatolie. Quant aux ouvrages grecs, ils privilégient souvent l'appellation «Musulmans» pour décrire les personnes avec lesquelles les Grecs sont en cohabitation en Anatolie ou en Grèce. L'appellation "Turcs » est fréquemment utilisée lorsqu'il s'agit de nommer les soldats adversaires ou les membres de l'autre communauté avec lesquels ils n'ont pas de relations pacifiques. 
17 En dehors de la dénomination liée aux identités ethniques et religieuses, certains livres grecs et turcs donnent largement place aux mots " ami » et « voisin " pour décrire les membres de l'autre communauté avec lesquels ils avaient des relations de voisinage, d'amitié ou de commerce. A titre d'exemple, dans le livre Iki Vatan Yorgunları composé de 59 témoignages des échangés turcs de première génération, nous comptons 17 fois l'utilisation du mot «ami » et "voisin » pour décrire les Grecs : « Nous nous entendions très bien avec nos voisins grecs. Ma mère avait des voisines grecques qu'elle fréquentait très souvent et moi j'avais Sofia comme meilleure amie » (Özsoy, 2003 : 75).

Le mot «ennemi » est souvent employé pour décrire les personnes avec lesquelles ils n'ont pas de relations directes mais plutôt lorsqu'ils se retrouvent dans le même endroit en raison de la guerre. D'autres livres préfèrent privilégier les adjectifs «bon » et « mauvais » à la place « d'ami » ou « ennemi ». Le Bon Turc ou le Bon Grec désigne dans la plupart des cas celui qui aide le membre de l'autre communauté en cas de danger ou de situation conflictuelle. Malgré plusieurs actes violents des Turcs contre les Grecs cités dans la plupart des témoignages, des citations à propos de «Bons Turcs » ne manquent pas dans les livres grecs à l'instar de celle de «Bons Grecs» dans les ouvrages turcs : « Nos voisins turcs ne nous ont jamais fait mal. Au contraire, ils nous ont caché des soldats turcs » (Kücük Asya Araştırmaları Merkezi, 2004: 99); «Dans notre village près de Kavala, les Turcs et les Grecs cohabitaient. Les deux peuples avaient de bonnes relations, à tel point qu'un voisin grec avait protégé mon grand-père contre les bandes armées (grecques)» (Özsoy, 2014 : 94). Il s'agit donc d'une solidarité entre les Grecs et les Turcs compatriotes: "Les Grecs locaux de Mytilène nous ont protégé contre les Grecs d'Anatolie qui sont venus dans notre île » (Özsoy, $2003: 51$ ).

Le Bon Turc/Grec et le Mauvais Turc/Grec sont souvent présents dans le même récit de vie : « Dans notre village, il existait de bons Grecs mais aussi de mauvais » (Özsoy, 2014 : 10). Parfois, l'existence de soldats turcs qui essayent d'anéantir les Grecs de la région n'empêche pas non plus la présence des Bons Turcs qui aident les Grecs : « Les Turcs du village de Hacı Ömer étaient nos compatriotes, ils connaissaient très bien mon père (...) ils nous ont toujours aidés et nourris quand nous avions faim » (Andreadis, 1999 : 79).

En raison de la situation de guerre, la transformation d'ami à ennemi est souvent évoquée dans les livres grecs et turcs: "En Crète, là où tout le monde vivait en paix et en tranquillité, la situation s'est inversée : les assassinats et violences ont rempli la vie des Chrétiens et des Musulmans (de l'île)» (Yorulmaz, 2010 : 43) ; "Les Turcs étaient nos amis, maintenant ils ne sont que nos ennemis » (Kücük Asya Araştırmaları Merkezi, 2004 : 50) ; «La situation s'est aggravée. Le nombre des voleurs a augmenté et nos femmes étaient harcelées (par les Turcs). Jusqu'à ce jour, nous n'avions pas vécu de tels événements» (Tsolakidis, 2007: 79); «Les gens que je connais depuis toujours commencent à ne plus me saluer, me parler. Et quand ils parlent, ils ne disent pas de bonnes choses : leur parole sent la poudre » (Yorulmaz, 2004 : 31) ; «L'armée d'occupation grecque nous traitait amicalement au début de l'occupation, elle est devenue violente après avoir signé l'armistice avec l'Empire ottoman » (Andıç, 2004 : 12).

Dans certains ouvrages turcs, pour décrire le Mauvais Grec, le mot « infidèle » (en Turc Gavur) est assez fréquemment utilisé (notamment dans le livre d'Aladağ, ce mot a été employé 36 fois) : «Où est-elle l'armée ottomane? Pourquoi elle ne vient pas nous sauver de ces infidèles?» (Andıç, 2004: 17); "Ces infidèles ont vécu avec notre tolérance pendant des années mais regarde ce qu'ils font maintenant! Ils sont ingrats... » (Aladağ, 1999 : 20). Les Turcs qui refusaient l'appellation de leurs amis grecs comme infidèles 
étaient aussi considérés par les autres turcs comme des traitres et des infidèles (Aladağ, 1999 : 21). Nous rencontrons l'utilisation du mot infidèle par les Turcs contre les Turcs qui ne savaient pas parler le Turc (car leur langue maternelle était le Grec) au moment de leur arrivée en Turquie : «En raison du fait que nous parlions le Grec, on nous appelait infidèles » (Özsoy, 2014 : 28) ; « Les habitants locaux de Tuzla (à İstanbul) nous ont rejeté au début de notre installation dans leur quartier. Ils nous appelaient infidèles car nous parlions le grec. » (Özsoy, 2003 : 93).

\section{La mémoire des migrations}

Les migrations des Grecs d'Anatolie vers la Grèce et celles des Turcs de la Grèce vers la Turquie ont eu de nombreuses conséquences sur les personnes concernées. Même si la migration obligatoire a eu plus d'effets destructeurs pour les Grecs que pour les Turcs, le traumatisme du déracinement et les difficultés d'intégration dans une nouvelle société ont marqué les mémoires des deux peuples à jamais. Le reflet de la mémoire des migrations dans les littératures grecque et turque a plusieurs traits communs comme la tristesse de quitter les territoires d'origine et le sentiment d'appartenance territoriale et s'exprime de diverses manières.

En premier lieu, le chagrin partagé entre les communautés turque et grecque en raison de l'obligation de quitter leurs terres natales attire l'attention. Dans plusieurs livres, le partage de la douleur de la séparation des terres natales et la haine commune contre les responsables de la décision de l'échange des populations sont exprimés : « les Turcs et les Grecs ont pleuré ensemble et banni ceux qui ont causé cet échange des populations » (Kücük Asya Araştırmaları Merkezi, 2004 : 202). Dans certains livres grecs, les auteurs attirent l'attention sur le fait que les Turcs étaient touchés du départ obligatoire de leurs voisins grecs car ils considéraient que l'Anatolie était non seulement leur territoire mais aussi celui des Grecs d'Anatolie : «Les gens ont dû quitter les terres de leurs ancêtres et leurs maisons à cause d'une décision arbitraire (l'échange des populations); ils se sont séparés de leurs compatriotes avec lesquels ils étaient proches comme des frères malgré leurs appartenances religieuses différentes » (Tsolakidis, $2007: 1$ ).

En deuxième lieu, nous remarquons dans les livres grecs et turcs que le lieu d'origine, malgré l'exil, ont gardé leur appellation de " patrie »: «Drama (en Grèce) est ma vraie patrie, quant à la Turquie, elle est ma deuxième patrie » (Özsoy, $2014: 120)$; «J'ai quitté l'Asie mineure, ma patrie, quand j'avais 15 ans. Le souvenir de ma patrie reste toujours très vif » (Kücük Asya Araştırmaları Merkezi, 2004 : 91) ; « Ici (en Grèce) notre situation est bien mais dans notre patrie, notre vie en Asie mineure était meilleure qu'ici » (Kücük Asya Araştırmaları Merkezi, 2004 : 192).

En troisième lieu, les Grecs d'Anatolie et les Turcs de Grèce ont rencontré plusieurs problèmes dès leur arrivée dans leur nouvelle patrie. Les problèmes d'adaptation culturelle et linguistique, le chômage, la pauvreté et le rejet social sont les problèmes les plus fréquemment cités dans les récits de vie : «Qu'est-ce qu’on a gagné en venant ici (en Grèce) ? Si on n'avait pas quitté l'Anatolie, on serait déjà morts donc sauvés; mais ici le danger c'est la pauvreté, la misère, la maladie et les conditions de vie inhumaines ». (Molivyatis, $2005:$ 153) ; «Comment est-il possible qu'on nous (les Turcs) envoie en Turquie alors que nous ne parlons même pas la langue et en échange, les Grecs d'Anatolie 
sont envoyés en Grèce malgré le fait qu'ils ne parlent que le Turc ?» (Lozan Mübadilleri Vakfi, 2009 : 227).

L'inquiétude de s'installer dans des nouveaux territoires définis désormais comme la nouvelle patrie est présente dans la plupart des témoignages turcs: "Nous sommes différents des populations originaires de ces terres (...) On avait quitté notre belle patrie (en Grèce) pour venir dans ces endroits étrangers (en Turquie) (Lozan Mübadilleri Vakfi, 2009 : 289) ; « l'Anatolie, même si c'est ma mère-patrie, c'est un endroit inconnu. Aller làbas en quittant ma Crète là où je suis né et j'ai grandi ? Pour moi, la patrie est ici (Crète) » (Yorulmaz, 2004: 95). Néanmoins, certains sont heureux d'avoir vécu l'échange des populations car ils pensent que cette migration était plus que nécessaire pour assurer la sécurité des peuples: "Nous souhaitions un échange entre les populations (grecque et turque) car il faut que chacune (des populations) vive sous le drapeau de sa nation " (Özsoy, 2003 : 46) ; « Heureusement qu'il y a eu l'échange des populations. Ici (en Grèce), nous ne fermons pas nos portes quand nous dormons. Nous n'avons plus peur des Turcs. Nous sommes en sécurité » (Kücük Asya Araştırmaları Merkezi, 2004 : 195).

En quatrième lieu, le manque des «anciennes patries» et la référence aux «patries d'autrefois » sont très présents dans les romans et les récits de vie : «L'envie de retourner vers les terres où j'ai grandi m'a déchiré le cœur pendant soixante-cinq ans de ma vie » (Tsolakidis, 2007: 44). Ayse, le personnage principal du roman intitulé Selanik, Kahpe Selanik, une fois arrivée de Thessalonique en Turquie, ne voulait rien entendre à propos de la Grèce car elle n'arrivait pas à oublier ce qu'elle avait souffert pendant les années de guerre. (Andıç, $2004: 36$ ) Mais quelques années après, le manque de sa ville natale devient de plus en plus fort et aucune ville ne lui faisait sentir comme la sienne à part Thessalonique (Andiç, 2004 : 42). Dans son livre Felegin Çemberinde, Yordanidou raconte l'histoire d'une famille d'Istanbul migrée obligatoirement en Grèce, elle attire aussi l'attention sur le manque du passé et de la patrie ainsi que l'impossibilité de comparer les nouvelles et les anciennes patries, ces dernières étant gravées à jamais dans le cœur des personnes concernées (Yordanidou, 1998: 174). Les échangés et les réfugiés essayent d'apaiser le manque des patries par divers moyens, comme une réunion entre amis, une discussion à propos de leur passé : « Mes parents parlaient souvent de la patrie et parfois ils se réunissaient avec les autres (échangés de Grèce) pour discuter de leurs anciens quartiers, amis et souvenirs (de la Grèce)» (Lozan Mübadilleri Vakfl,, 2009: 55); «Le propriétaire du restaurant était très sympathique ou je le trouvais comme ça car il était d'Istanbul. Nous échangions des blagues en Turc et nous éclations de rire " (Tsolakidis, 2007 : 209). C'est l'exil même qui rendait la Patrie plus présente dans ces territoires nouveaux.

\section{Conclusion}

Nous pouvons conclure que les récits de vie grecs et turcs examinés partagent certains traits majeurs en même temps qu'ils présentent des différences notoires de perception de la guerre et de ses conséquences. Cependant, les différences n'excluent pas le fait que les Grecs et les Turcs sont tous "des enfants de la guerre», comme l'auteur Yorulmaz les nomme dans son livre Savasın Çocukları et que leurs mémoires ont été façonnées par les difficultés et le traumatisme de la guerre et des migrations causées par les années de guerre. La lecture de leurs récits de vie nous permet de saisir l'histoire commune de la Grèce et de la Turquie d'un point de vue différent de celui de la lecture des histoires 
nationales. Ainsi, cette lecture pourrait permettre de comprendre « l'autre " puisqu'elle donne l'occasion, par ses différences, de percevoir d'autres réalités souvent négligées, et par ses similarités, d'approcher les peuples qui ont partagé les mêmes sentiments.

\section{BIBLIOGRAPHIE}

ALADAĞ, Ertuğrul (1999). Maria, Göç Acısl. Istanbul : Belge.

ANDIÇ, Fuat (2004). Selanik, Kahpe Selanik. Istanbul : Eren.

ANDREADIS, Yorgo (1999). Tolika "Bacikam Al Ben"i. Istanbul : Belge.

BILICI, Faruk (2004). « La littérature grecque contemporaine traduite en turc : un capital de sympathie ", Cahiers balkaniques, 33, disponible le 07/04/2015.

URL : http://ceb.revues.org/3198

KÜÇÜK ASYA ARAşTIRMALARI MERKEZI (2004). Göç, Rumların Anadolu'dan Mecburi Ayrıllşı 1919-1923.

Istanbul : Iletişim.

LOZAN MÜBADILLERI VAKFI (2009). Mübadele Öyküleri. Istanbul : LMV Yayınları.

MILLAS, Herkül (2005). Türk ve Yunan Romanlarında Öteki ve Kimlik. Istanbul: Iletisim.

MOLIVYATIS, Agapi (2005). On Günün Günlügü. Istanbul: Albatros.

ÖZsoY, İskender (2014). Mübadelenin Yas Kardesleri. Istanbul: Bağlam.

ÖzsoY, İskender (2003). Iki Vatan Yorgunları: Mübadele Acısını Yaşayanlar Anlatıyor. Istanbul: Bağlam. SOTIRIYU, Dido (2013). Benden Selam Soyle Anadolu'ya. Istanbul: Can Sanat.

TSOLAKIDIS, Kostas (2007). Belki Bir Gün Dönerim. Istanbul: Literatür.

XANTHOULIS, Yiannis (2005). Bahçede bir Türk. Istanbul: Sistem.

YORDANIDU, Maria (1998). Felegin Çemberinde. Istanbul: Belge.

YORULMAZ, Ahmet (2004). Savasın Çocukları. Istanbul: Remzi.

YORULMAZ, Ahmet (2010). Ulya. Istanbul : Kirmızı Kedi.

\section{RÉSUMÉS}

Les années des guerres entre la Turquie et la Grèce et les migrations qui les ont suivies, ont marqué et façonné la mémoire des Grecs et des Turcs et par conséquent les récits de vie des témoins de cette époque. Ce travail est consacré à l'étude des récits de vie des Grecs et des Turcs dans le but de saisir les similarités et les différences de la période conflictuelle dans la littérature en Turquie et en Grèce.

The years of war between Greece and Turkey and the migrations that followed have influenced the memory of Greek and Turkish people and by consequence their life stories. This work is 
focused on the study of the life stories of Greek and Turkish people in order to understand the similarities and the differences in the period of conflict as presented in the literature in Greece and Turkey.

INDEX

Mots-clés : guerre, mémoire, récit de vie, Turquie, Grèce

Keywords : war, memory, life story, Turkey, Greece

\section{AUTEURS}

\section{GÖKÇE BAYINDIR GOULARAS}

Université Yeditepe

gokce.bayindir@yeditepe.edu.tr

\section{AYŞE BETÜL NUHOĞLU}

Université Yeditepe

betulnuhoglu@gmail.com 